\title{
Early-Onset Type 2 Diabetes as a Risk Factor for End-Stage Renal Disease in Patients With Diabetic Kidney Disease
}

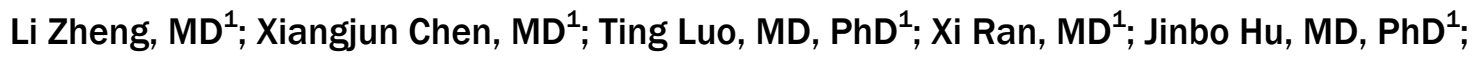 \\ Qingfeng Cheng, MD, $\mathrm{PhD}^{1}$; Shumin Yang, MD, $\mathrm{PhD}^{1}$; Jinshan $\mathrm{Wu}, \mathrm{MD}, \mathrm{PhD}^{1}$; Qifu Li, MD, PhD ${ }^{1}$; \\ Zhihong Wang, MD, $\mathrm{PhD}^{1}$
}

\begin{abstract}
Accessible Version: www.cdc.gov/pcd/issues/2020/20_0076.htm
Suggested citation for this article: Zheng L, Chen X, Luo T, Ran X, Hu J, Cheng Q, et al. Early-Onset Type 2 Diabetes as a Risk Factor for End-Stage Renal Disease in Patients With Diabetic Kidney Disease. Prev Chronic Dis 2020;17:200076. DOI: https:// doi.org/10.5888/pcd17.200076.
\end{abstract}

\section{PEER REVIEWED}

\section{Summary}

What is already known on this topic?

Compared with the type 2 diabetes of typical onset in middle age or older, type 2 diabetes with early age of onset has a higher risk of diabetesrelated complications.

What is added by this report?

In patients with diabetic kidney disease (DKD), the prevalence of endstage renal disease (ERSD) in the early-onset group was twofold higher than it was in the late-onset group. Early-onset diabetes is an independent risk factor for ESRD in patients with DKD.

What are the implications for public health practice?

Prevention strategies for diabetic complications should emphasize closer attention to patients with DKD and early-onset diabetes. More stringent metabolic targets should be applied to delay DKD progression.

\section{Abstract}

\section{Introduction}

Compared with the typical onset of type 2 diabetes in middle age or older, type 2 diabetes with early age of onset has a higher risk of diabetes-related complications. It is unclear whether the early age of diabetes diagnosis would affect the development of endstage renal disease (ESRD) in patients with diabetic kidney disease (DKD) who are at higher risk of ESRD.

\begin{abstract}
Methods
We enrolled 1,111 type 2 diabetes patients with DKD in this study. We used the age at diabetes diagnosis of younger than 40 years to define early-onset diabetes and 40 years or older to define late-onset diabetes. Medical history, anthropometry, and laboratory indicators were documented. ESRD was defined by estimated glomerular filtration rate (eGFR) of less than $15 \mathrm{~mL} / \mathrm{min} / 1.73 \mathrm{~m}^{2}$ or dialysis. Logistic regression analysis was used to explore the association between early-onset diabetes and ESRD.
\end{abstract}

\section{Results}

Early-onset diabetes patients had a longer diabetes duration, higher body mass index, and worse blood lipid metabolism profile. Compared with late-onset diabetes patients, patients with earlyonset diabetes had a prevalence of ESRD that was twofold higher $(9.2 \%$ vs $4.3 \% ; P=.009)$. Univariate analysis showed that earlyonset diabetes was a risk factor for ESRD in patients with DKD $(P$ $<.05)$. In multivariate logistic regression analysis, even after adjusting for sex, traditional metabolic factors, drug factors, and diabetes duration, the risk of ESRD in patients with early-onset diabetes was still 3.58 -fold higher than in subjects with late-onset (95\% CI, 1.47-8.74; $P=.005$ ).

\section{Conclusions}

In patients with DKD, early-onset type 2 diabetes is an independent risk factor of ESRD.

\section{Introduction}

End-stage renal disease (ESRD) is a global health problem with increasing prevalence (1). Diabetic kidney disease (DKD) is the leading cause of ESRD, accounting for approximately $50 \%$ of cases in developed countries worldwide $(2,3)$. The progress of DKD to ESRD negatively affects the treatment of diseases and increases the economic burden of individuals and societies $(4,5)$. 
Therefore, early detection of risk factors for DKD progressing to ESRD is a promising strategy for reducing related mortality and social economic burden.

Duration of diabetes has been considered a strong factor in the development of vascular complications of type 2 diabetes (6). Some studies have found that a subset of patients with longer diabetes duration are characterized by an earlier age of diabetes onset. Compared with patients with type 2 diabetes of typical onset in middle age or older, patients with type 2 diabetes with early age of onset (early-onset diabetes) have poorer metabolic control; higher risks for chronic kidney disease, retinopathy, and neuropathy (7-9); higher incidence of nonfatal cardiovascular disease (10); and higher standardized mortality (11).

Whether early-onset diabetes will affect DKD progressing to ESRD is still unknown. One study showed that early-onset diabetes has higher rates of macroalbuminuria (12). The appearance of macroalbuminuria has been viewed as the beginning of progressive renal function loss and impending failure or ESRD. Therefore, early-onset diabetes may promote the occurrence of ESRD. A prospective study found that younger onset of diabetes (onset age $<20$ years) increased the risk of ESRD, even after adjusting for age and sex (7). However, that study focused on type 2 diabetes in adolescents. It is unknown whether this conclusion is consistent in adultonset type 2 diabetes patients with DKD who are at higher risk of ESRD. Therefore, we investigated 1,111 type 2 diabetes patients with DKD to determine the relationship between early-onset diabetes and the risk of ESRD.

\section{Methods}

\section{Study design and study population}

This is a retrospective cross-sectional study from The First Affiliated Hospital of Chongqing Medical University in China. We enrolled 1,111 inpatients (686 men and 425 women) with diagnosed type 2 diabetes with DKD who were in the hospital from January 1,2014 , to June 25,2018 . They were screened from the electronic medical record system based on inclusion and exclusion criteria. The inclusion criteria were the following:

- type 2 diabetes meeting the 1999 World Health Organization's diagnostic criteria for type 2 diabetes (13): 1) fasting plasma glucose (FPG; fasting defined as no caloric intake for at least $8 \mathrm{~h}$ ) of $126 \mathrm{mg} / \mathrm{dL}$ or higher $(7.0$ $\mathrm{mmol} / \mathrm{L}$ or higher); or 2) 2-h plasma glucose value of $200 \mathrm{mg} / \mathrm{dL}$ or higher (11.1 mmol/L or higher) during a 75-g oral glucose tolerance test; or 3) a patient with classic symptoms of increased thirst, urine volume, unexplained weight loss, and a random plasma glucose of $200 \mathrm{mg} / \mathrm{dL}$ or higher (11.1 $\mathrm{mmol} / \mathrm{L}$ or higher);

- aged 18 years or older;
- have at least 1 previous inpatient medical record for diabetes; and

- diagnosed with DKD, defined as meeting at least 1 of the following (14): 1) random urinary microalbumin creatinine ratio (UACR) was $30 \mathrm{mg} / \mathrm{g}$ or higher creatinine in at least 2 of 3 tests; or 2) estimated glomerular filtration rate (eGFR) was $60 \mathrm{~mL} / \mathrm{min} / 1.73 \mathrm{~m}^{2}$ or less.

The exclusion criteria were 1) patients without information about diabetes duration; 2) patients with half or more missing data on all key variables; 3 ) pregnant or lactating women; 4) patients with type 1 diabetes or other special types of diabetes; 5) patients definitely diagnosed with other types of chronic renal diseases such as IgA nephropathy or membranous nephropathy; 6) patients with urinary tract obstruction, urinary tract injury, urinary tract infection, or other conditions affecting urinary albumin; 7) patients who need long-term glucocorticoid treatment of other chronic diseases; and 8) patients with immune dysfunction, severe infection, or malignant tumor history. Patients were defined as having early-onset diabetes and late-onset diabetes based on the age at diagnosis of diabetes of less than 40 years or 40 years or older, respectively $(15,16)$. The selection of 40 years as a cutoff was based on the use of similar age strata (20-39 years, $40-59$ years, and 60-79 years) in the latest International Diabetes Federation estimates of world diabetes prevalence (17). Previous studies have used 40 years as an age threshold for young-onset diabetes $(15,18)$, especially the 2 large sample surveys in Mainland China and Hong Kong $(10,12)$.

\section{Clinical procedures}

We reviewed clinical information including age, sex, history of diabetes (including duration of diabetes and age at first diagnosis of diabetes), smoking and drinking history, the history of concomitant diseases or diabetes complications, and medication history. All patients underwent physical anthropometry measurements including weight, height, systolic blood pressure (SBP), diastolic blood pressure (DBP), and body mass index (BMI), which was calculated by dividing weight in kilograms by height in meters squared. Laboratory assessments, including plasma glucose levels, glycosylated hemoglobin $\left(\mathrm{HbA}_{1 \mathrm{c}}\right)$, total cholesterol (TC), triglyceride (TG), high density lipoprotein cholesterol (HDL-C), low density lipoprotein cholesterol (LDL-C), serum creatinine ( $\mathrm{sCr}$ ), urinary creatinine, and albumin were done in our hospital. Details of the measurement method were given in our previous study (19). UACR was calculated by urinary albumin/creatinine ratio. eGFR was estimated by using the Modification of Diet in Renal Disease Study equation (men: eGFR $=186 \times \mathrm{sCr}^{-1.154} \times$ year $^{-0.203}$; women: $\mathrm{eGFR}=186 \times \mathrm{sCr}^{-1.154} \times$ year $\left.^{-0.203 \times 0.724}\right)(20)$. ESRD was defined as dialysis or by eGFR lower than $15 \mathrm{~mL} / \mathrm{min} / 1.73 \mathrm{~m}^{2}(12)$.

The opinions expressed by authors contributing to this journal do not necessarily reflect the opinions of the U.S. Department of Health and Human Services, the Public Health Service, the Centers for Disease Control and Prevention, or the authors' affiliated institutions. 


\section{Statistical analysis}

Statistical analysis was performed by using the Statistical Package for Social Science (SPSS) version 20.0 (IBM). Data were expressed as mean (standard deviation [SD]) when the sample distribution is approximately normal. If the data were not a normal distribution model after testing with a quantile-quantile map, we used median (interquartile range) for expression. Continuous variables between 2 groups were compared by using the $t$ test or Mann-Whitney $U$ test. Categorical variables were reported as frequencies (proportions), and $\chi^{2}$ tests were used for group comparisons. We conducted univariate logistic analysis to investigate risk factors for ESRD in patients with DKD and multivariate logistic regression analysis to determine whether early-onset diabetes could be an independent risk factor for ESRD. Four models were analyzed to adjust for confounding factors, and the included variables were significant indicators in the univariate logistic analysis and indicators suggested by previous studies. Model 1 was adjusted for sex. Model 2 was further adjusted for BMI, blood pressure, blood lipids, $\mathrm{HbA}_{1 \mathrm{c}}$, and history of smoking, drinking, and hypertension in addition to the variable in model 1 . Model 3 was adjusted for drug factors on the basis of model 2. Model 4 further adjusted for duration of diabetes in addition to the variables in model 3. Bilateral $P$ values less than .05 were considered significant.

\section{Results}

\section{Clinical features of patients}

The mean (SD) age of the 1,111 patients with DKD was 63.6 (10.8) years, with a mean (SD) diabetes duration of 11.9 (7.2) years. A total of $152(13.7 \%)$ patients had early-onset type 2 diabetes. Compared with late-onset diabetes patients, early-onset diabetes patients were more likely to be male, younger, have had earlier onset, have had a longer diabetes duration, and have had a history of smoking and drinking. They had a higher BMI, had higher DBP, worse blood lipid profile (higher TC, TG, and LDLC) and blood glucose metabolism (higher $\mathrm{HbA}_{1 \mathrm{c}}$ concentrations), and had higher UACR and eGFR. Patients with early onset diabetes were less likely to be treated with oral hypoglycemic drugs, antihypertensive drugs, and renin-angiotensin system (RAS) blockers. Although there were higher proportions of insulin therapy and lower proportions of lipid-lowering drugs in the earlyonset diabetes group, these differences were not significant (Table $1)$.

Patients with early-onset diabetes had higher prevalence of ESRD $(9.2 \%)$ than patients with late-onset diabetes $(4.3 \%)(P=.009)$. Furthermore, we observed that the mean (SD) age of diabetes onset was significantly younger in patients with ESRD than those without ESRD (47.5 [11.8] years vs $52.4[11.0]$ years, $P=.001)$ (Figure), and the percentage of early-onset diabetes in patients with ESRD was higher than it was in those without ESRD $(25.5 \%$ vs $13.1 \%, P=.009$, Pearson $\chi^{2}$ test).

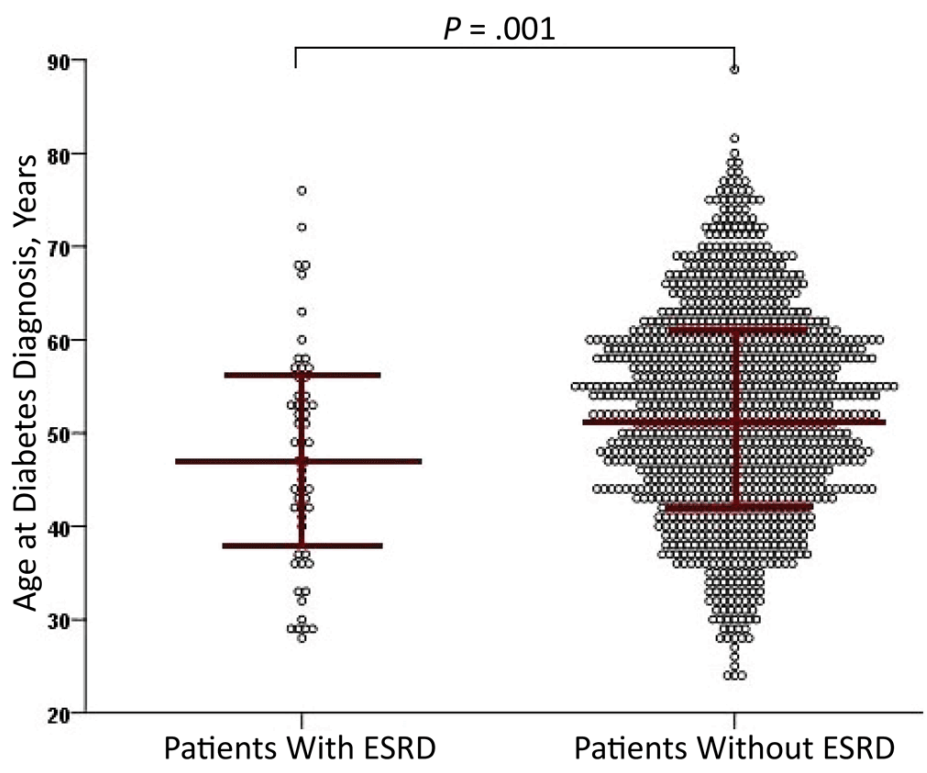

Figure. Age at diabetes diagnosis in 1,111 patients with or without end-stage renal disease (ESRD), The First Affiliated Hospital of Chongqing Medical University, China, 2014-2018. Circles represent patients; red lines represent mean and standard deviation.

\section{Risk factors for ESRD in patients with DKD}

Univariate logistic analysis was used to identify the risk factors for ESRD in patients with DKD. Risk factors for ESRD were earlyonset type 2 diabetes (odds ratio [OR], 2.27 [95\% CI, 1.21-4.28]; $P=.011$ ), duration of diabetes (OR, 1.07 [95\% CI, 1.03-1.11]; $P<$ $.001)$, history of hypertension (OR, 3.81 [95\% CI, 1.62-8.98]; $P=$ $.002)$, SBP (OR, 1.02 [95\% CI, 1.01-1.03]; $P=.001)$, use of insulin therapy (OR, 3.16 [95\% CI, 1.53-6.53]; $P=.002$ ), and use of antihypertensive drugs (OR, 3.78 [95\% CI, 1.76-8.08]; $P=.001)$. However, we found that $\mathrm{HbA}_{1 \mathrm{c}}$ concentrations, use of oral hypoglycemic drugs, and use of RAS blockers were protective factors for ESRD, and age, sex, blood lipids, BMI, and use of lipid-lowering drugs were not related to the development of ES$\mathrm{RD}$ (Table 2).

Multivariate logistic analysis was conducted to adjust for confounding factors. We found that patients with early-onset type 2 diabetes had 2.3-fold (95\% CI, 1.2-4.4) higher risk of ESRD compared with patients with late-onset type 2 diabetes after adjustment for sex (our model 1), and the difference was significant (Table 3). Further adjustment for other metabolic factors (BMI, SBP,

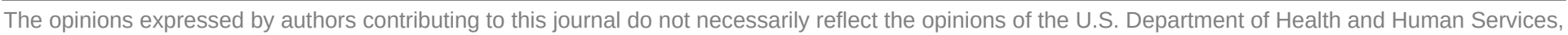
the Public Health Service, the Centers for Disease Control and Prevention, or the authors' affiliated institutions. 
DBP, TC, TG, LDL-C, HDL-C, HbA $1 \mathrm{c}$, smoking history, drinking history, and hypertension history) in patients with early-onset type 2 diabetes was associated with 4.5-fold (95\% CI, 2.0-10.0) increased risk for ESRD compared with patients with late-onset type 2 diabetes (our model 2) (Table 3). The increased risk of ESRD was only slightly attenuated after further adjustment for drug factors (oral hypoglycemic drugs, insulin therapy, antihypertensive drugs, RAS blockers, lipid-lowering drugs) (our model 3) (OR, 4.5; 95\% CI, 1.9-10.6) (Table 3). After further adjustment for duration of diabetes, the risk of ESRD decreased somewhat, but the overall correlation remained significant (our model 4) (OR, 3.6; 95\% CI, 1.5-8.7) (Table 3).

\section{Discussion}

In our study, we demonstrated for the first time that early-onset diabetes is associated with the risk of ESRD in the patients with DKD. This finding remains significant after adjusting for potential confounding factors such as duration of diabetes, sex, traditional metabolic factors, and drug factors, suggesting that earlyonset diabetes is an independent risk factor for ESRD in patients with DKD.

The onset age of type 2 diabetes has been decreasing, and the prevalence of early-onset type 2 diabetes is increasing rapidly in both developed and developing countries $(21,22)$. The International Diabetes Federation estimated that roughly 63 million young adults aged 20 to 39 years had type 2 diabetes worldwide in 2013; early-onset diabetes accounted for $16 \%$ of total adults with type 2 diabetes (23). In our study, early-onset type 2 diabetes accounted for $13.7 \%$ of all recruited patients. With the increased prevalence of early-onset diabetes, the incidence of diabetic complication such as cardiovascular and cerebrovascular diseases, chronic kidney disease, and even ESRD is gradually increasing (7-10). A longitudinal population-based study in the United States reported that onset of type 2 diabetes in participants younger than age 20 years was associated with a nearly fivefold increase in the incidence of ESRD between 25 and 54 years of age compared with a later onset of type 2 diabetes (7). Data from a prospective cohort study in Singapore also showed that early-onset type 2 diabetes was associated with a substantially higher risk of progressive chronic kidney disease compared with late-onset type 2 diabetes (24). These studies agree with our data showing that early-onset type 2 diabetes patients have increased risk of ESRD. However, a cross-sectional study of the Joint Asia Diabetes Evaluation cohort study found that although there was a higher proportion of ESRD in the earlyonset diabetes group, this difference was not significant (12). In addition, in the United States study (7), Pavkov et al also said that the effect of youth-onset type 2 diabetes increasing the risk of ESRD will disappear after adjusting for age, sex, diabetes duration, and other factors. Pavkov and colleagues conducted their research in the Pima Indian population, which might be quite different from our hospital-based study. Also, Pavkov and colleagues limited the analysis of ESRD to young and middle-aged Pima Indians, because data were insufficient to compute adjusted incidence rates in the youth-onset group older than age 55 years and in the olderonset group younger than age 25 years. This lack of data might introduce selection bias. Besides, our research object was patients with DKD and the population spectrum is narrower, which is also a possible reason for the inconsistent results.

The mechanisms by which early-onset diabetes increases the risk of DKD progression have not been fully clear. Several epidemiologic studies have found that the increased risk of ESRD associated with early-onset type 2 diabetes can be attributed to the prolonged duration of diabetes and subsequent deterioration of metabolic status. For example, a cross-sectional survey in 77 tertiary hospitals in China found that early-onset diabetes greatly increased the prevalence of microvascular diseases compared with late-onset diabetes after adjusting for age, sex, and traditional risk factors, but the difference was not significant after adjusting for disease duration (9). Prolonged diabetes duration might lead to long-term exposure to hyperglycemic conditions, which might lead to reduced $\beta$-cell function, increased oxidative stress, and activation of the RAS (25), and then promote the progression of diabetes complications and increase the risk of ESRD. However, our study found that early-onset diabetes is an independent risk factor for ESRD, even after adjusting for duration of diabetes. This may be related to genetic variation and strong family history of diabetes. Kong et al found that early-onset diabetes had a strong genetic predisposition, which could be mainly linked to $\beta$-cell function (26). Other investigations showed that HNF4 $\alpha$ protein expression was repressed and T allele rs3760106 variation of PRKCB1 and the $G$ allele rs2575390 variation were correlated with the occurrence of DKD in patients with early-onset diabetes $(27,28)$. Protein kinase C- $\beta$ encoded by PRKCB1 gene is closely related to the occurrence of macroalbuminuria, eGFR decline, and ESRD $(29,30)$. The above evidence suggests that diabetes duration and genetic susceptibility play an important role in the occurrence of early-onset diabetes and the development of vascular complications. Other known mechanisms include socioeconomic and psychological-behavioral. Whether other mechanisms exist needs further investigation.

Our study has the following strengths. First, the relationship between early-onset type 2 diabetes and ESRD has been reported for the first time in the DKD population. With the increased prevalence of early-onset diabetes, our research has the important clinical implication that we need to pay more attention to the sub-

The opinions expressed by authors contributing to this journal do not necessarily reflect the opinions of the U.S. Department of Health and Human Services, the Public Health Service, the Centers for Disease Control and Prevention, or the authors' affiliated institutions. 
set of DKD patients with early-onset diabetes, and apply more stringent metabolic targets to delay DKD progression. Second, we studied a large sample. Third, our study minimizes the effect of duration or other risk factors by using statistical adjustments.

Some limitations of the study are worth mentioning. First, our study was cross-sectional. Prospective cohort studies to explore the cause-effect association between early-onset type 2 diabetes and ESRD in patients with DKD are needed. Second, patients were selected among hospitalized patients in a single center, which might lead to a selection bias. Also, the results need to be verified by a larger sample size from a multicenter population. In addition, we cannot know the precise onset age of type 2 diabetes because of the insidious onset of this disease. The observed onset age may be older than the real onset age.

Our study provides information on the effect of the age of diagnosis of diabetes on the development of ESRD in patients with DKD. Compared with type 2 diabetes of typical onset in middleaged and elderly patients, patients with early-onset diabetes have poorer blood lipids and blood glucose status and a higher prevalence of proteinuria and ESRD. These perspectives highlight the growing imperative to direct attention toward early-onset type 2 diabetes and for effective interventions to be applied before middle age.

\section{Acknowledgments}

Li Zheng and Xiangjun Chen should be considered as joint first author.

We thank the Chongqing Medical University for their support toward publishing this article. We also thank the staff and patients of The First Affiliated Hospital of Chongqing Medical University for their contributions during the study. Finally, we thank Xinlu Lv, Ying Tang, Qinying Zhao, Yangmei Zhou, Wenjin Luo, and Weiling Zhao for helping with data collection for the study.

Li Zheng and Xiangjun Chen designed the study and wrote the manuscript. Ting Luo and Jinbo Hu oversaw the data collection and contributed to the study design. Li Zheng and Xi Ran conducted the data analysis. Xinlu Lv, Ying Tang, Qinying Zhao, Yangmei Zhou, Wenjin Luo, and Weiling Zhao contributed to the data collection. Jinshan Wu and Qingfeng Cheng contributed to the writing of the manuscript. Shichao Han and Shumin Yang edited the manuscript. Zhihong Wang and Qifu Li are the guarantors of this work and, as such, had full access to all the data in the study and take responsibility for the integrity of the data and the accuracy of the data analysis.
The authors declare that they have no conflict of interest with the contents of this article.

This work was supported by the National Natural Science Foundation of China (grant numbers 81670785 and 81970720), Technological Innovation and Application Development Project of Chongqing (grant no. cstc2019iscx-msxm0546), Chongqing Science and Health Joint Medical Research Project (grant no. 2018GDRC004), and High-end Medical Talents of Middle-aged and Young People in Chongqing (Yuweiren [2015] 49).

This study was approved by Ethical Committee of The First Affiliated Hospital of Chongqing Medical University. Informed consent was obtained from all participants.

\section{Author Information}

Corresponding Author: Zhihong Wang, The First Affiliated Hospital of Chongqing Medical University, Yuzhong District, Chongqing, China. Telephone: +86-023-89011552. E-mail: towzh713@126.com.

Author Affiliations: ${ }^{1}$ Department of Endocrinology, The First Affiliated Hospital of Chongqing Medical University, Chongqing, China.

\section{References}

1. Levey AS, Coresh J. Chronic kidney disease. Lancet 2012; 379(9811):165-80.

2. Afkarian M, Zelnick LR, Hall YN, Heagerty PJ, Tuttle K, Weiss NS, et al. Clinical manifestations of kidney disease among US adults with diabetes, 1988-2014. JAMA 2016; 316(6):602-10.

3. Reutens AT. Epidemiology of diabetic kidney disease. Med Clin North Am 2013;97(1):1-18.

4. KDOQI. KDOQI clinical practice guidelines and clinical practice recommendations for diabetes and chronic kidney disease. Am J Kidney Dis 2007;49(2Suppl 2):S12-154.

5. Nichols GA, Vupputuri S, Lau H. Medical care costs associated with progression of diabetic nephropathy. Diabetes Care 2011;34(11):2374-8.

6. Nanayakkara N, Ranasinha S, Gadowski A, Heritier S, Flack JR, Wischer N, et al. Age, age at diagnosis and diabetes duration are all associated with vascular complications in type 2 diabetes. J Diabetes Complications 2018;32(3):279-90.

7. Pavkov ME, Bennett PH, Knowler WC, Krakoff J, Sievers ML, Nelson RG. Effect of youth-onset type 2 diabetes mellitus on incidence of end-stage renal disease and mortality in young and middle-aged Pima Indians. JAMA 2006;296(4):421-6.

The opinions expressed by authors contributing to this journal do not necessarily reflect the opinions of the U.S. Department of Health and Human Services, the Public Health Service, the Centers for Disease Control and Prevention, or the authors' affiliated institutions. 
8. Wong J, Molyneaux L, Constantino M, Twigg SM, Yue DK. Timing is everything: age of onset influences long-term retinopathy risk in type 2 diabetes, independent of traditional risk factors. Diabetes Care 2008;31(10):1985-90.

9. Li L, Ji L, Guo X, Ji Q, Gu W, Zhi X, et al. Prevalence of microvascular diseases among tertiary care Chinese with early versus late onset of type 2 diabetes. J Diabetes Complications 2015;29(1):32-7.

10. Huo X, Gao L, Guo L, Xu W, Wang W, Zhi X, et al. Risk of non-fatal cardiovascular diseases in early-onset versus lateonset type 2 diabetes in China: a cross-sectional study. Lancet Diabetes Endocrinol 2016;4(2):115-24.

11. Al-Saeed AH, Constantino MI, Molyneaux L, D’Souza M, Limacher-Gisler F, Luo C, et al. An inverse relationship between age of type 2 diabetes onset and complication risk and mortality: the impact of youth-onset type 2 diabetes. Diabetes Care 2016;39(5):823-9.

12. Yeung RO, Zhang Y, Luk A, Yang W, Sobrepena L, Yoon $\mathrm{KH}$, et al. Metabolic profiles and treatment gaps in youngonset type 2 diabetes in Asia (the JADE programme): a crosssectional study of a prospective cohort. Lancet Diabetes Endocrinol 2014;2(12):935-43. Erratum in Lancet Diabetes Endocrinol 2014;2(12):e17.

13. World Health Organization. Definition, diagnosis and classification of diabetes mellitus and its complications. Report of a WHO Consultation. Part 1: diagnosis and classification of diabetes mellitus. Geneva $(\mathrm{CH})$ : World Health Organization; 1999.

14. Introduction: standards of medical care in diabetes - 2018. Diabetes Care 2018;41(Suppl 1):S1-2.

15. Luk AO, Lau ES, So WY, Ma RC, Kong AP, Ozaki R, et al. Prospective study on the incidences of cardiovascular-renal complications in Chinese patients with young-onset type 1 and type 2 diabetes. Diabetes Care 2014;37(1):149-57.

16. Song SH. Emerging type 2 diabetes in young adults. Adv Exp Med Biol 2012;771:51-61.

17. Shaw JE, Sicree RA, Zimmet PZ. Global estimates of the prevalence of diabetes for 2010 and 2030. Diabetes Res Clin Pract 2010;87(1):4-14.

18. Song SH, Hardisty CA. Early onset type 2 diabetes mellitus: a harbinger for complications in later years - clinical observation from a secondary care cohort. QJM 2009; 102(11):799-806.

19. Hu J, Yang S, Zhang A, Yang P, Cao X, Li X, et al. Abdominal obesity is more closely associated with diabetic kidney disease than general obesity. Diabetes Care 2016; 39(10):e179-80.
20. Ma YC, Zuo L, Chen JH, Luo Q, Yu XQ, Li Y, et al. Modified glomerular filtration rate estimating equation for Chinese patients with chronic kidney disease. J Am Soc Nephrol 2006; 17(10):2937-44.

21. Lascar N, Brown J, Pattison H, Barnett AH, Bailey CJ, Bellary $\mathrm{S}$. Type 2 diabetes in adolescents and young adults. Lancet Diabetes Endocrinol 2018;6(1):69-80.

22. Harron KL, Feltbower RG, McKinney PA, Bodansky HJ, Campbell FM, Parslow RC. Rising rates of all types of diabetes in south Asian and non-south Asian children and young people aged 0-29 years in West Yorkshire, U.K., 1991-2006. Diabetes Care 2011;34(3):652-4.

23. International Diabetes Federation. IDF diabetes atlas. 6th edition. Brussels (BE): International Diabetes Federation, 2013. http://www.diabetesatlas.org/resources/previouseditions.html. A ccessed April 1, 2017.

24. Liu JJ, Liu S, Gurung RL, Ang K, Tang WE, Sum CF, et al. Risk of progressive chronic kidney disease in individuals with early-onset type 2 diabetes: a prospective cohort study. Nephrol Dial Transplant 2020;35(1):115-21.

25. Liguori A, Abete P, Hayden JM, Cacciatore F, Rengo F, Ambrosio G, et al. Effect of glycaemic control and age on lowdensity lipoprotein susceptibility to oxidation in diabetes mellitus type 1. Eur Heart J 2001;22(22):2075-84.

26. Kong X, Xing X, Zhang X, Hong J, Yang W. Early-onset type 2 diabetes is associated with genetic variants of $\beta$-cell function in the Chinese Han population. Diabetes Metab Res Rev 2020; 36(2):e3214.

27. Niehof M, Borlak J.HNF4 $\alpha$ and the Cachannel $<\mathrm{i}>\mathrm{TRPC} 1</ \mathrm{i}>$ are novel disease candidate genes in diabetic nephropathy. Diabetes 2008;57(4):1069-77.

28. Ma RC, Tam CH, Wang Y, Luk AO, Hu C, Yang X, et al. Genetic variants of the protein kinase $C-\beta 1$ gene and development of end-stage renal disease in patients with type 2 diabetes. JAMA 2010;304(8):881-9.

29. Noh H, King GL. The role of protein kinase $\mathrm{C}$ activation in diabetic nephropathy. Kidney Int Suppl 2007;72(106):S49-53.

30. Ishii H, Jirousek MR, Koya D, Takagi C, Xia P, Clermont A, et al. Amelioration of vascular dysfunctions in diabetic rats by an oral PKC $\beta$ inhibitor. Science 1996;272(5262):728-31.

\footnotetext{
The opinions expressed by authors contributing to this journal do not necessarily reflect the opinions of the U.S. Department of Health and Human Services, the Public Health Service, the Centers for Disease Control and Prevention, or the authors' affiliated institutions.
} 


\section{Tables}

Table 1. Clinical Characteristics of Patients With Early-Onset and Late-Onset Type 2 Diabetes, The First Affiliated Hospital of Chongqing Medical University, China, 2014-2018

\begin{tabular}{|c|c|c|c|c|}
\hline \multirow[b]{2}{*}{ Characteristic } & \multirow[b]{2}{*}{ Overall $(\mathrm{N}=1,111)$} & \multicolumn{3}{|c|}{ Onset } \\
\hline & & Early $(n=152)$ & Late $(n=959)$ & $P$ Value \\
\hline Age, mean (SD), y & $63.6(10.8)$ & $48.4(8.4)$ & $66(9.1)$ & $<.001$ \\
\hline $\begin{array}{l}\text { Age of diabetes onset, mean (SD), } \\
y\end{array}$ & $52.1(11.0)$ & $34.6(3.8)$ & $54.9(9.1)$ & $<.001$ \\
\hline Duration of diabetes, mean (SD), y & $11.9(7.2)$ & $13.6(8.0)$ & $11.6(7.1)$ & .001 \\
\hline Male, n (\%) & $686(61.7)$ & $116(76.3)$ & $570(59.4)$ & $<.001$ \\
\hline Hypertension, $\mathrm{n}(\%)^{\mathrm{a}}$ & $763(69.2)$ & $79(53.0)$ & $684(71.8)$ & $<.001$ \\
\hline Smoking, n (\%) & $531(47.8)$ & 99 (65.1) & $432(45.0)$ & $<.001$ \\
\hline Drinking, n (\%) & $436(39.2)$ & $86(56.6)$ & $350(36.5)$ & $<.001$ \\
\hline Body mass index ${ }^{b}$, mean (SD) & $25.1(3.6)$ & $25.8(3.8)$ & $25.0(3.6)$ & .013 \\
\hline $\begin{array}{l}\text { Systolic blood pressure, mean } \\
\text { (SD), mmHg }\end{array}$ & $143.7(22.5)$ & $140.9(24.6)$ & $144.1(22.1)$ & .010 \\
\hline $\begin{array}{l}\text { Diastolic blood pressure, mean } \\
\text { (SD), mmHg }\end{array}$ & $79.6(13.6)$ & $86.6(13.6)$ & $78.4(13.2)$ & $<.001$ \\
\hline $\begin{array}{l}\text { Total cholesterol, mean (SD), } \\
\mathrm{mmol} / \mathrm{L}\end{array}$ & $4.2(1.3)$ & $4.5(1.4)$ & $4.1(1.2)$ & $<.001$ \\
\hline $\begin{array}{l}\text { Total triglyceride, mean (SD), } \\
\mathrm{mmol} / \mathrm{L}\end{array}$ & $2.1(1.9)$ & $2.7(2.4)$ & $2.0(1.7)$ & $<.001$ \\
\hline $\begin{array}{l}\text { High density lipoprotein } \\
\text { cholesterol, mean (SD), mmol/L }\end{array}$ & $1.1(0.4)$ & $1.0(0.3)$ & $1.1(0.4)$ & .21 \\
\hline $\begin{array}{l}\text { Low density lipoprotein cholesterol, } \\
\text { mean (SD), mmol/L }\end{array}$ & $2.5(1.1)$ & $2.7(1.1)$ & $2.4(1.0)$ & .009 \\
\hline $\mathrm{HbA}_{1 \mathrm{c}}$, mean (SD), \% & $9.1(2.4)$ & $9.5(2.4)$ & $9.0(2.4)$ & .04 \\
\hline $\begin{array}{l}\text { Urinary microalbumin creatinine } \\
\text { (Cr) ratio, median (interquartile } \\
\text { range), } \mathrm{mg} / \mathrm{g} \mathrm{Cr}\end{array}$ & $194.1(69.1-753.7)$ & $221.2(77.6-1,250.0)$ & $184.1(67.2-695.0)$ & .03 \\
\hline $\begin{array}{l}\text { Estimated glomerular filtration } \\
\text { rate, mean (SD) }\end{array}$ & $67.9(34.6)$ & $80.3(43.0)$ & $66.0(32.7)$ & $<.001$ \\
\hline Oral hypoglycemic drugs, n (\%) & $640(57.6)$ & $74(48.7)$ & $566(59.0)$ & .02 \\
\hline Insulin therapy, $\mathrm{n}(\%)^{\mathrm{a}}$ & $691(62.9)$ & $101(67.8)$ & $590(62.1)$ & .18 \\
\hline Antihypertensive drugs, $\mathrm{n}(\%)^{\mathrm{a}}$ & $672(61.6)$ & $72(48.3)$ & $600(63.7)$ & $<.001$ \\
\hline RAS blockers, $\mathrm{n}(\%)^{\mathrm{a}}$ & $408(37.4)$ & $41(27.5)$ & $367(39.0)$ & .007 \\
\hline Lipid-lowering drugs, n (\%) & $216(19.4)$ & $22(14.5)$ & $194(20.2)$ & .11 \\
\hline End stage renal disease, $\mathrm{n}(\%)$ & $55(5.0)$ & $14(9.2)$ & $41(4.3)$ & .009 \\
\hline
\end{tabular}

Abbreviations: $\mathrm{Cl}$, confidence interval; $\mathrm{HbA}_{1 \mathrm{c}}$, glycated hemoglobin; RAS blockers, renin-angiotensin system blockers, including angiotensin converting enzyme inhibitors and angiotensin receptor antagonists.

a Data were the effective percentage; the number of patients with missing data has been subtracted from the denominator.

${ }^{\mathrm{b}}$ Calculated as weight in kilograms divided by height in meters squared. 
Table 2. Univariate Logistic Regression Analysis for End Stage Renal Disease in Patients With Diabetic Kidney Disease, The First Affiliated Hospital of Chongqing Medical University, China, 2014-2018

\begin{tabular}{|c|c|c|}
\hline Variable & Odds Ratio (95\% Confidence Interval) & $P$ value \\
\hline Age, y & $1.00(0.97-1.02)$ & .80 \\
\hline Sex, male vs female & $1.00(0.57-1.75)$ & .99 \\
\hline Onset, early vs late & $2.27(1.21-4.28)$ & .011 \\
\hline Duration of diabetes, $y$ & $1.07(1.03-1.11)$ & $<.001$ \\
\hline History of hypertension & $3.81(1.62-8.98)$ & .002 \\
\hline $\mathrm{HbA}_{1 \mathrm{c}}, \%$ & $0.72(0.62-0.85)$ & $<.001$ \\
\hline Total cholesterol, $\mathrm{mmol} / \mathrm{L}$ & $1.09(0.89-1.33)$ & .42 \\
\hline Total triglyceride, $\mathrm{mmol} / \mathrm{L}$ & $0.88(0.72-1.08)$ & .22 \\
\hline Low density lipoprotein cholesterol, $\mathrm{mmol} / \mathrm{L}$ & $1.15(0.91-1.45)$ & .26 \\
\hline Systolic blood pressure, $\mathrm{mmHg}$ & $1.02(1.01-1.03)$ & .001 \\
\hline Diastolic blood pressure, $\mathrm{mmHg}$ & $0.99(0.97-1.01)$ & .46 \\
\hline Body mass index ${ }^{a}$ & $1.01(0.93-1.09)$ & .89 \\
\hline Use of oral hypoglycemic drugs & $0.19(0.10-0.36)$ & $<.001$ \\
\hline Use of insulin therapy & $3.16(1.53-6.53)$ & .002 \\
\hline Use of antihypertensive drugs & $3.78(1.76-8.08)$ & .001 \\
\hline Use of RAS blockers & $0.37(0.18-0.73)$ & .005 \\
\hline Use of lipid-lowering drugs & $1.43(0.76-2.67)$ & .27 \\
\hline
\end{tabular}

Abbreviations: $\mathrm{HbA}_{1 \mathrm{c}}$, glycated hemoglobin; RAS blockers, renin-angiotensin system blockers, including angiotensin converting enzyme inhibitors and angiotensin receptor antagonists.

${ }^{a}$ Calculated as weight in kilograms divided by height in meters squared. 
Table 3. Odds Ratio of Early-Onset Type 2 Diabetes for End Stage Renal Disease in Patients With Diabetic Kidney Disease, The First Affiliated Hospital of Chongqing Medical University, China, 2014-2018

\begin{tabular}{|l|r|r|}
\hline Model & \multicolumn{1}{|c|}{ Odds Ratio (95\% Confidence Interval) } \\
\hline Model 1, early vs late onset $^{\mathrm{a}}$ & $\mathbf{P}$ value \\
\hline Model 2, early vs late onset $^{\mathrm{b}}$ & $\mathbf{0 1 1}$ \\
\hline Model 3, early vs late onset $^{\mathrm{c}}$ & $<.3(1.2-4.4)$ & $4.5(2.0-10.0)$ \\
\hline Model 4, early vs late onset $^{\mathrm{d}}$ & $\mathbf{< . 0 0 1}$ \\
\hline
\end{tabular}

${ }^{\text {a }}$ Adjusted for sex.

${ }^{\mathrm{b}}$ Further adjusted for traditional metabolic factors, including body mass index, systolic blood pressure, diastolic blood pressure, total cholesterol, total triglyceride, high density lipoprotein cholesterol, low density lipoprotein cholesterol, glycated hemoglobin, smoking history, drinking history, and hypertension history in addition to sex.

${ }^{c}$ Further adjusted for use of oral hypoglycemic drugs, insulin, antihypertensive drugs, renin-angiotensin system blockers (including angiotensin converting enzyme inhibitors and angiotensin receptor antagonists), and lipid-lowering drugs in addition to the variables in model 2.

${ }^{\mathrm{d}}$ Further adjusted for the duration of diabetes in addition to the variables in model 3. 